\title{
AIP
}

\section{Giant magnetoelectric torque effect and multicoupling in two phases ferromagnetic/piezoelectric system}

Zengping Xing, Kai Xu, Guangyu Dai, Jiefang Li, and Dwight Viehland

Citation: Journal of Applied Physics 110, 104510 (2011); doi: 10.1063/1.3662912

View online: http://dx.doi.org/10.1063/1.3662912

View Table of Contents: http://scitation.aip.org/content/aip/journal/jap/110/10?ver=pdfcov

Published by the AIP Publishing

\section{Articles you may be interested in}

Resonance magnetoelectric effects in a layered composite under magnetic and electrical excitations

J. Appl. Phys. 112, 014103 (2012); 10.1063/1.4733466

Theoretical model for geometry-dependent magnetoelectric effect in magnetostrictive/piezoelectric composites J. Appl. Phys. 111, 124513 (2012); 10.1063/1.4729832

Piezoelectric single crystal langatate and ferromagnetic composites: Studies on low-frequency and resonance magnetoelectric effects

Appl. Phys. Lett. 100, 052901 (2012); 10.1063/1.3679661

Energy harvesting properties of all-thin-film multiferroic cantilevers

Appl. Phys. Lett. 99, 203506 (2011); 10.1063/1.3662037

Extremely low frequency response of magnetoelectric multilayer composites

Appl. Phys. Lett. 86, 102901 (2005); 10.1063/1.1881784

MIT LINCOLN

LABORATORY CAREERS

Discover the satisfaction of innovation and service

to the nation
- Space Control

- Air \& Missile Defense

- Communications Systems \& Cyber Security

- Intelligence, Surveillance and

Reconnaissance Systems

- Advanced
Electronics
- Tactical Systems
- Homeland
Protection
- Air Traffic Control

LINCOLN LABORATORY

MassachusetTs Institute of TeChNOLOgY

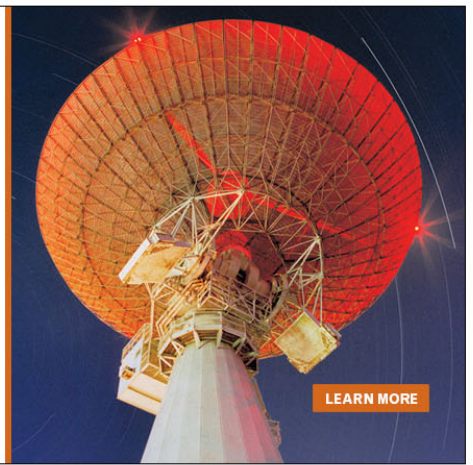




\title{
Giant magnetoelectric torque effect and multicoupling in two phases ferromagnetic/piezoelectric system
}

\author{
Zengping Xing, ${ }^{1,2, a)}$ Kai Xu, ${ }^{1}$ Guangyu Dai, ${ }^{1}$ Jiefang $\mathrm{Li}^{2}{ }^{2}$ and Dwight Viehland ${ }^{2}$ \\ ${ }^{1}$ Key Laboratory of Magnetic Materials and Devices, Ningbo Institute of Material Technology \\ and Engineering, Chinese Academy of Sciences, Ningbo, People's Republic of China, 315201 \\ ${ }^{2}$ Virginia Tech, Blacksburg, Virginia 24061, USA
}

(Received 25 August 2011; accepted 17 October 2011; published online 29 November 2011)

\begin{abstract}
The physical mechanism of a non-magnetostrictive magnetoelectric (ME) effect was revealed and designated as the ME torque (MET) effect. Experimental results showed that the MET effect could be huge; a simple MET device could achieve giant ME voltage coefficients of $100 \mathrm{~V} / \mathrm{cm}$.Oe at $1 \mathrm{~Hz}$ and $2100 \mathrm{~V} / \mathrm{cm}$.Oe at the first order resonant frequency. These are the highest reported $\mathrm{ME}$ coefficients in a bulk device ever. We then proposed the multicoupling ME effect, which comes from the interaction of magnetostriction, magnetic torque, and piezoelectricity, and rewrite the ME constitutive tensor equation. The abnormal phenomenon in the (1-3) structure ME thin film that T-L mode might bring larger ME coupling than L-L mode was successfully explained from the multicoupling concept. These researches have extended the giant ME effect from the traditional magnetostrictive/piezoelectric system to a common ferromagnetic/piezoelectric system, and gave more choices to scientists/engineers for constructing the giant ME device. (C) 2011 American Institute of Physics. [doi:10.1063/1.3662912]
\end{abstract}

\section{BACKGROUND}

The magnetoelectric (ME) effect is characterized by an induced dielectric polarization in response to an applied magnetic field $(\mathrm{H})$, or by an induced magnetization in response to an applied electric field (E). ${ }^{1}$ It can be evaluated by a parameter designated as the $\mathrm{ME}$ voltage coefficient $\alpha_{\mathrm{ME}}$, which can be written as $\alpha_{\mathrm{ME}}=\delta \mathrm{E} / \delta \mathrm{H}=\left(\delta \mathrm{V}_{\mathrm{i}} / \mathrm{t}\right) / \delta \mathrm{H}$ (where $\mathrm{H}$ is the applied magnetic field, $\mathrm{E}$ and $\mathrm{V}_{\mathrm{i}}$ are the electric field and voltage induced across the piezoelectric (PE) layer, and $\mathrm{t}$ is the thickness or more accurate the effective distance between the electrodes of the PE phase). ${ }^{2}$

The ME effect was proposed earlier by Curie in 1894 and discovered experimentally in $\mathrm{Cr}_{2} \mathrm{O}_{3}$ by Astrov in $1960{ }^{3,4}$ After that, numerous single phase ME materials were reported, however, direct coupling between spin and dipole in atomic scale is very difficult, which makes the ME effect in single phase quite small. ${ }^{5-9}$ Scientists then turned to the consideration of structural composites; the ME particle composite was first reported by Philips Research Lab in Holland in the mid1970 s. This composite approach combines magnetostrictive (MS, or piezomagnetic (PM)) and PE phases, which interact via elastic force. ${ }^{10}$ Later on, many successful researches were carried out. ${ }^{11-14}$ However, the ME effect in a particle composite, although much larger than that in a single phase, is still much smaller than that predicted by theory due to some technical limitations. ${ }^{1}$ Until 2001, the giant ME effect was first reported in laminated composites consisting of MS and PE layers, although both giant MS and PE materials had been found for decades. ${ }^{15}$ Since then, the researches on MS-PE laminate composite developed quickly and successfully in theory and engineering. ${ }^{16-23}$ However, the applications are

${ }^{\text {a)} E l e c t r o n i c ~ m a i l: ~ x i n g z p @ n i m t e . a c . c n . ~}$ always limited by the materials properties, price, and the forming technology. Some people then tried to find new giant MS materials, new forming technologies, and some began to seek new mechanisms. ${ }^{24,25}$

Although the ME effect could appear in the ferromagnetic (FM)/PE two phase system from the symmetry point of view, giant ME contribution of the two phase system are always accredited to the MS-PE interaction. ${ }^{1,23,26}$ This might comes from the intuitive understanding of the intimate contact coupling of the MS and PE phase, however, another coupling has been overlooked for years. In the following section, we will introduce this ME coupling which comes from magnetic-torque (MT). This non-MS ME coupling will give us more consideration on the ME effect contribution; multicoupling from magnetostriction, magnetic-torque, and piezoelectricity. Giant $\mathrm{ME}$ devices will not be limited to the MS-PE two phase system, but to a broader system constructed from the FM-PE phase.

\section{MECHANISM AND EXPERIMENTS}

The MT $\tau$ to a FM phase in an applied magnetic field $\mathbf{B}$ can be calculated from the classic electrodynamics as ${ }^{27}$

$$
\tau=\mathbf{m} \times \mathbf{B}=(\mathbf{M} \times \mathbf{B}) \cdot V,
$$

where $\mathbf{m}, \mathbf{M}$ are the magnetic moment and magnetization of the FM phase, respectively, and $V$ the phase's volume. This MT $\tau$ will try to line up the magnetic moment $\mathbf{m}$ and the external field $\mathbf{B}$. If one tried to keep $\mathbf{m}$ and $\mathbf{B}$ in different orientation by structural design or specific boundary condition, strain will occur inside the FM phase. If this strain is coupled to the PE phase, the electro-mechanical coupling inside the PE phase then induces a charge output. This is how and why the MT will contribute to the ME effect in a FM-PE two phase system. We designated the MT to the ME effect as ME 
(a)

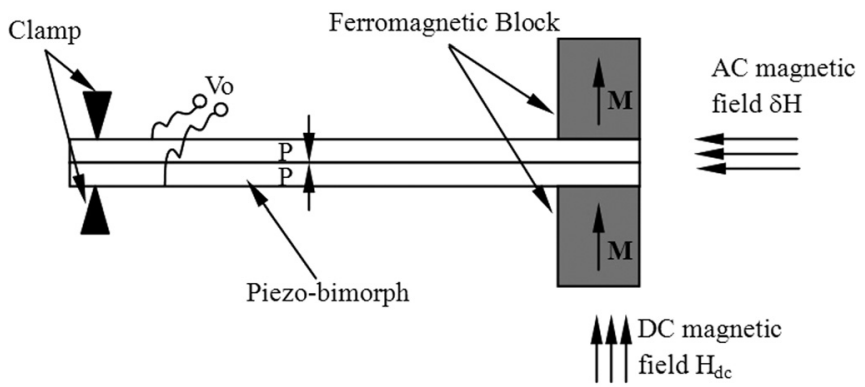

(b)

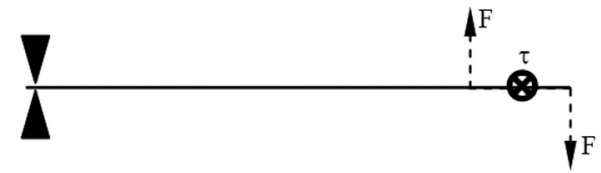

FIG. 1. (a) A recommended structure for the MT-PE coupling device; and (b) illustration of the working mechanism.

torque (MET) effect, and as a comparison, we designated the traditional MS-PE coupling ME effect as MES effect. From equation (1), we could evaluate the MET effect by

$$
\alpha_{\mathrm{MET}}=\kappa \cdot|\mathbf{M}| \cdot \sin \theta \cdot V / \mu_{0},
$$

where $\kappa$ is the MT-PE transfer function, $\theta$ the angular between $\mathbf{M}$ and $\mathbf{B}$, and $\mu_{0}$ the permeability of the free space.

From equation (2), one can see that the $\alpha_{\text {MET }}$ linearly increases with $\kappa, \mathrm{M}$, and $V$. The angle $\theta$ brings a spatial effect to the $\alpha_{\text {MET }}$, and a maximum value of $\alpha_{\text {MET }}$ could be achieved as it is equal to $90^{\circ}$. M, $V$, and $\theta$ could be easily controlled by the materials/device designers, however, $\kappa$ is quite a complex parameter; it is highly structural dependence. To increase $\kappa$, one should increase the bending mode MT-PE coupling due to the torque contribution. A simple
TABLE I. Magnetic unit samples.

\begin{tabular}{lccc}
\hline \hline No. & Material & Magnet shape & Magnet volume $\left(\mathrm{cm}^{3}\right)$ \\
\hline F1/N1 & Ferrite/NdFeB & $2 \times \phi 8 \times 3$ & 0.3 \\
F2/N2 & Ferrite/NdFeB & $2 \times \phi 8 \times 6$ & 0.6 \\
F3/N3 & Ferrite/NdFeB & $2 \times \phi 8 \times 9$ & 0.9 \\
F4/N4 & Ferrite/NdFeB & $2 \times \phi 8 \times 12$ & 1.2 \\
\hline \hline
\end{tabular}

piezo-bimorph structure, as recommended in Fig. 1(a), will help much. The apply DC magnetic field $\mathrm{H}_{\mathrm{dc}}$ helps to increase the magnetization $\mathrm{M}$ inside the ferromagnetic unit. A ferromagnetic unit with large residual flux density, such as $\mathrm{Fe}_{3} \mathrm{O}_{4}, \mathrm{NdFeB}$, AlniCo, Ticonal, SmCo, etc., will help to save the extra mechanism for producing the $\mathrm{H}_{\mathrm{dc}}$. If one tries a ferromagnetic unit with low residual flux density, such as cobalt, nickel, silicon-steel, conetic, permalloy, mumetal, metglass, etc., the $\mathrm{H}_{\mathrm{dc}}$ should be applied. The higher the saturate magnetic flux density Bs, the higher the MET effect could achieve.

To evaluate how strong the MET effect could be, we constructed MET devices with structure shown in Fig. 1(a). We used NdFeB (N35 type) or $\mathrm{Fe}_{3} \mathrm{O}_{4}$ Ferrite as the ferromagnetic units for saving the mechanism of producing the $\mathrm{H}_{\mathrm{dc}}$ due to their high residual flux density $(1.2 \mathrm{~T}$ and $0.3 \mathrm{~T}$, respectively), and they all have low magnetostriction. The sizes of the ferromagnetic units used in the experiments are shown in Table I. The piezo-bimorph was constructed by two pieces of PZT-850 (each $30 \times 6 \times 0.3 \mathrm{~mm}^{3}$ in size), whose layers were poled in opposite directions. The ferromagnetic units were attached to the tip of the PZT bimorph by use of a high strength epoxy. In Fig. 2(a), it can be seen that, with the mass of the magnetic units increase, the resonant frequency will decrease; this is because of the tip mass loading contribution. ${ }^{28}$ And the $\mathrm{NdFeB}$ has much higher

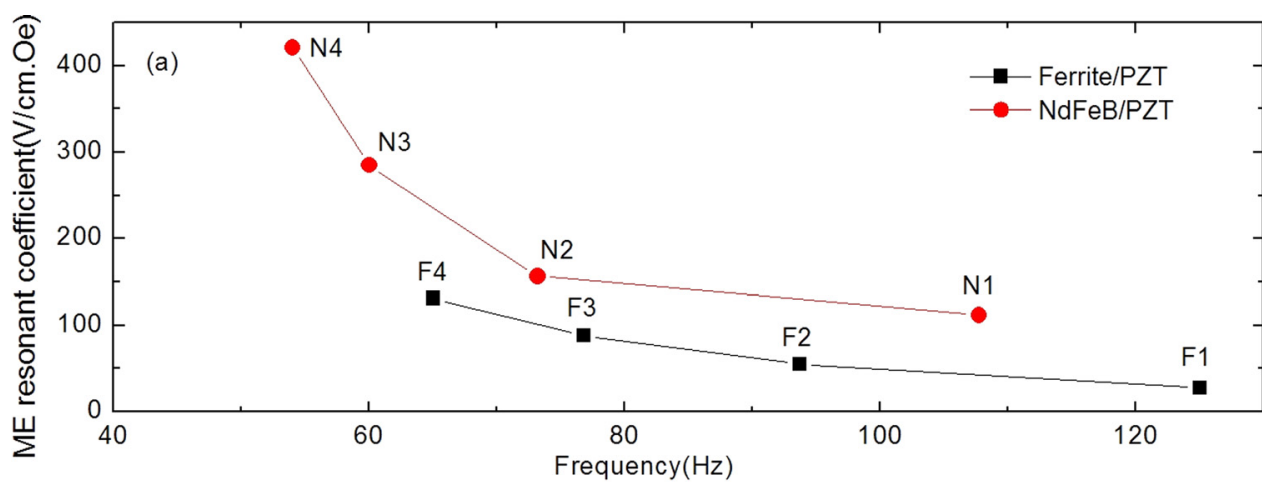

FIG. 2. (Color online) ME voltage coefficients for samples in Table I (a) at the first order bending resonant frequency; and (b) on the magnetic units volume $\mathrm{V}$

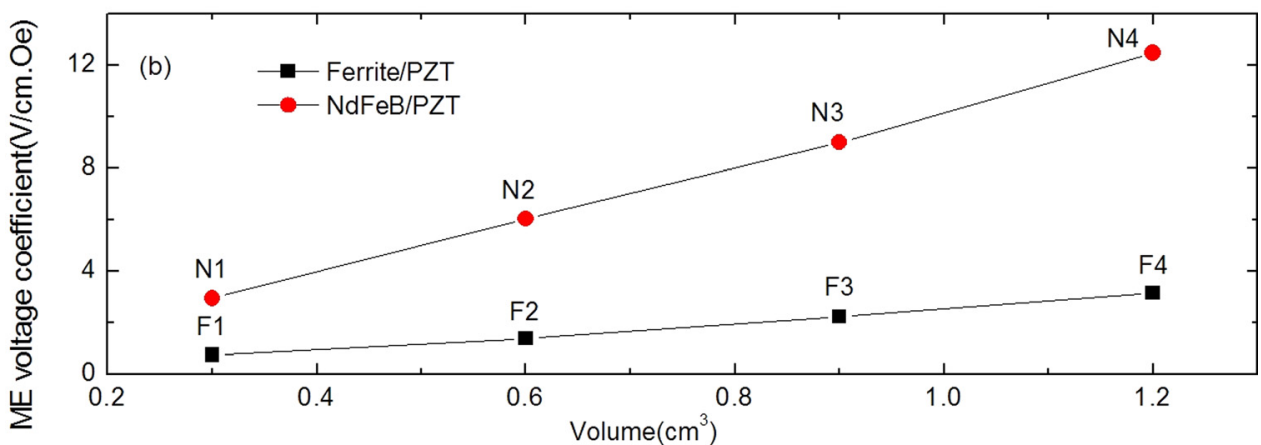
at $10 \mathrm{~Hz}$. 


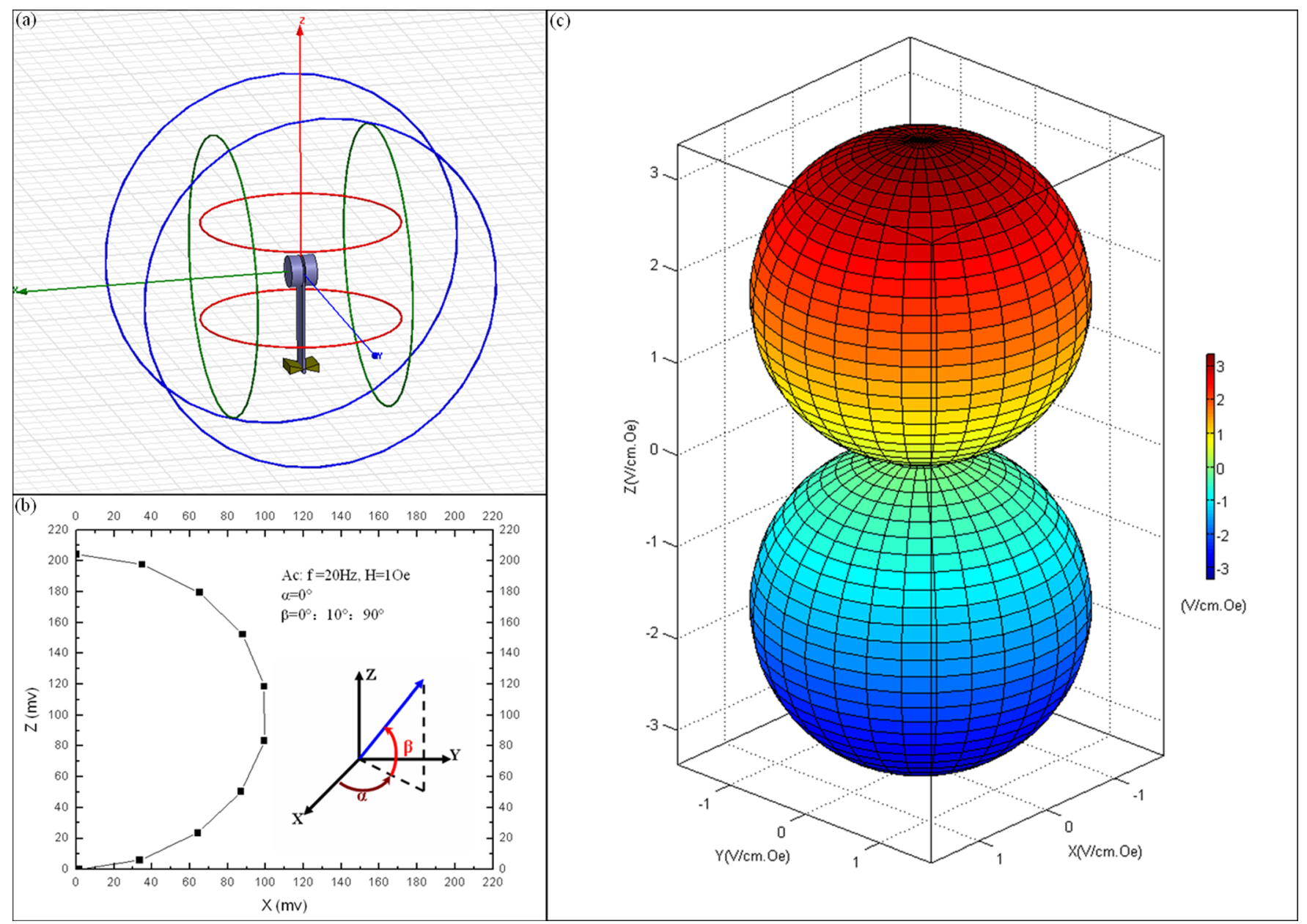

FIG. 3. (Color online) (a) Setup for spatial ME measurement; (b) ME effect in one quadrant plane; and (c) full spatial ME effect.

residual flux density than that of the $\mathrm{Fe}_{3} \mathrm{O}_{4}$ ferrite; which leads to a much higher MET effect at the resonant frequency for the same mass. Figure 2(b) supports equation (2) quite well: i) The ME voltage coefficients linearly increased with the volumes; ii) the slopes are 10.53 and 2.7 for the $\mathrm{NdFeB} /$ PZT samples and ferrite/PZT samples, separately, which coincided well with the ratio of their residual flux densities. The spatial MET effect could be evaluated by setting the MET device (N1) inside three Helmholtz coil pairs with each axial perpendicular to each other, as shown in Fig. 3(a). The experimental data for the ME effect were shown in Fig. 3 (b) for one quadrant plane $\left(\alpha=0^{\circ}, \beta=0: 10^{\circ}: 90^{\circ}\right)$ and Fig. 3(c) for full space. It can be seen that the ME effect is $\sin \theta$ dependence, which also fit equation (2) as well.

We also used two pieces of $\mathrm{NdFeB}$ (N42 Type, $\mathrm{Br} \approx 1.3 \mathrm{~T}$ ) with size of $50.8 \times 25.4 \times 3.2 \mathrm{~mm}^{3}$ each as the ferromagnetic units and did the same ME measurement. We acquired a figure of ME voltage coefficient as illustrated in Fig. 4. In this result, we acquired giant ME coefficients of $100 \mathrm{~V} / \mathrm{cm}$.Oe or $400 \mathrm{nC} / \mathrm{cm}$.Oe at a low frequency $(1 \mathrm{~Hz})$ and $2100 \mathrm{~V} / \mathrm{cm}$.Oe or $8.4 \mu \mathrm{C} / \mathrm{cm}$.Oe at the first order resonant bending frequency. These values are much larger than the highest value ever reported for a MS-PE device, which was $22 \mathrm{~V} / \mathrm{cm}$.Oe at the low frequency and $737 \mathrm{~V} / \mathrm{cm}$.Oe at the resonance. ${ }^{25,29}$ These results show that the MET effect could be huge and we should take the MET effect into the ME coupling consideration.

\section{MULTICOUPLING AND DISCUSSION}

Since the FM phase possesses the MT easily $(\theta \neq 0)$, the MET effect exists widely in multiferroics composite and should be taken into the ME coupling consideration. As the MET was taken into the consideration, the traditional MSPE coupling will then be extended to a multi-coupling of

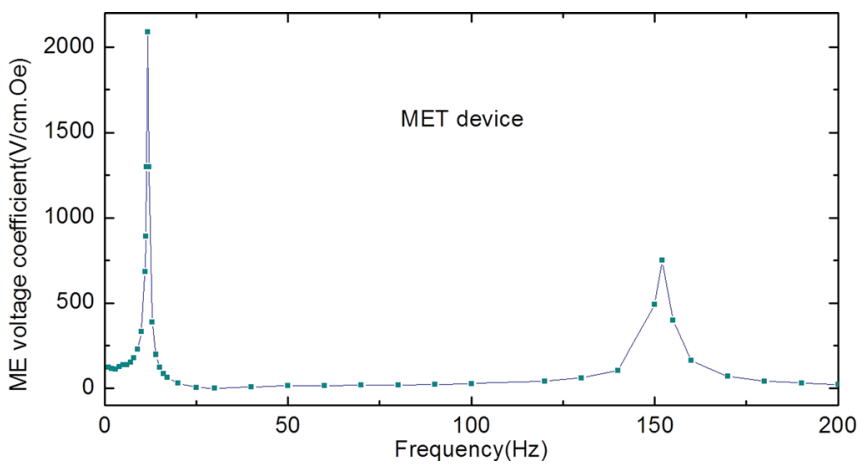

FIG. 4. (Color online) Frequency dependence of the ME voltage coefficient for the MET device. 
magnetostriction, magnetic-torque, and piezoelectricity, and the ME coefficient could be evaluated as

$$
\begin{gathered}
\partial P / \partial H=\alpha=k_{c} e^{m} e+k_{t} e^{T} e+\rho\left(e^{m}, e^{T}\right) e, \\
\partial P /\left.\partial H\right|_{e^{T}=0}=\alpha_{M E S}=k_{c} e^{m} e, \\
\partial P /\left.\partial H\right|_{e^{m}=0}=\alpha_{M E T}=k_{t} e^{T} e,
\end{gathered}
$$

where $k_{c}$ is the coupling factor of the MS and PE phase, $\mathrm{e}^{\mathrm{m}}$ and $\mathrm{e}$ are piezomagnetic and piezoelectric coefficients, respectively, $k_{t}$ is the coupling factor between the magnetictorque and PE phase, $e^{T}$ the magnetic-torque strain factor, $\rho\left(e^{m}, e^{T}\right)$ the correlation factor of the magnetic-torque and piezomagnetic to the strain.

For the case of $e^{T}=0$ (nonmagnetic torque case), equation (3a) becomes ( $3 \mathrm{~b})$, the same as the previous report, ${ }^{23}$ and the ME coupling will be a pure MS-PE coupling. For the case of $e^{m}=0$ (nonmagnetostrictive case), equation (3a) becomes $(3 \mathrm{c})$, and one can still achieve a giant ME effect by making full use of $e^{T}$. For a normal case that $e^{T}$ and $e^{m}$ coexist, giant MS-PE coupling does not mean a giant ME coefficient due to the fact that $e^{T}$ might play a contrary role, and giant MT-PE coupling does not mean giant ME effect either. Proper design should consider both MS and MT contribution. MT-PE coupling was considered mostly in Fig. 1(a); however, for multicoupling, Fig. 1(a) might not be a good choice because it might reduce the MS contribution. The design will be quite complicated as the multicoupling is taken into the consideration. As an example, let us consider a fundamental structure of clamped-free unimorph as show in Fig. 5. One can easily judge that $\delta \mathrm{H}_{1}$ helps the MS-PE coupling, while $\delta \mathrm{H}_{2}$ helps the MT-PE coupling. An optimized multi-coupling will require that the device is set to some angle to the detected magnetic field $\delta \mathrm{H}_{\mathrm{T}}$; and we designated this angle $\phi$ as optimized multi-coupling angle $\varphi_{\text {МОРт. }}$. It is a complex parameter that related to the materials properties, device structure and the boundary condition (clamp status, $\mathrm{H}_{\mathrm{DC}}$, etc.). The electric field $\mathrm{E}$ of the device can then be calculated as

$$
\begin{aligned}
E & =\alpha_{M E} \cdot H=\alpha_{M E T} \cdot H+\alpha_{M E S} \cdot H+\rho\left(\alpha_{M E T}, \alpha_{M E S}\right) H \\
& =T \cdot \sin \theta \cdot\left|B^{\prime}\right| \cdot|B| \cdot V / \mu_{0}+\alpha_{M E S} \cdot H+\rho\left(\alpha_{M E T}, \alpha_{M E S}\right) H .
\end{aligned}
$$

It is not that easy to determine the correlation factor $\rho$ due to the fact that the stress, strain, electric displacement, magnetic

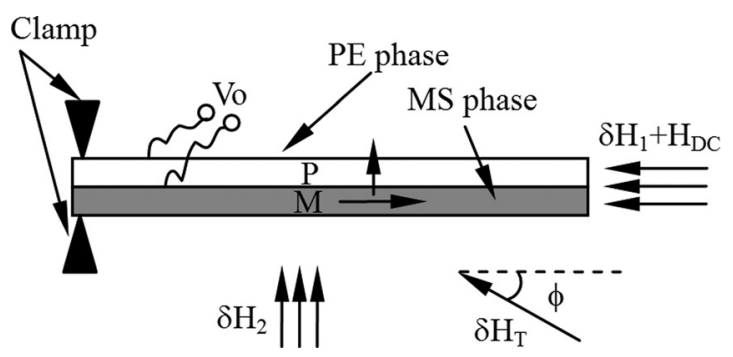

FIG. 5. Illustration of multicoupling for a unimorph cantilever. induction would coupling together, which makes it complex. It will be much better to evaluate $\alpha_{\mathrm{ME}}$ by writing the constitutive equation in tensor form for the magneticmechanical-electric coupling as follows:

$$
\begin{gathered}
\boldsymbol{\sigma}=c \mathbf{S}-e^{T} \mathbf{E}-\left(q^{T}+q_{M}^{T}\right) \mathbf{H}, \\
\mathbf{D}=e \mathbf{S}+\varepsilon \mathbf{E}+\alpha \mathbf{H}+\mathbf{P}_{\mathbf{S}}, \\
\mathbf{B}=\left(q+q_{M}\right) \mathbf{S}+\alpha^{T} \mathbf{E}+\mu \mathbf{H}+\mathbf{M}_{\mathbf{S}},
\end{gathered}
$$

where $\boldsymbol{\sigma}, \mathbf{S}, \mathbf{D}, \mathbf{E}, \mathbf{B}$, and $\mathbf{H}$ are stress, strain, electric displacement, electric field, magnetic induction, and magnetic field tensor, respectively, $c, \varepsilon$, and $\mu$ are the stiffness, dielectric constant, and permeability tensor separately; $e, q, q_{M}$ are, respectively, piezoelectric, piezomagnetic, magnetic-torque (MT) coefficient tensor; $\mathbf{P}_{\mathbf{S}}, \mathbf{M}_{\mathbf{S}}$ are the spontaneous polarization and magnetization tensor separately; and $\alpha$ is the $\mathrm{ME}$ coefficient. The superscript $T$ denotes the transpose of the tensor. The solution for static and dynamic status could refer to Refs. 12 and 30, respectively. However, finite element method will help much as the FM-PE system become complicated.

Figure 5 is the fundamental for nano-pillar ME composites grow on a substrate. As the multi-coupling ME effect is considered, the anomaly that the ME coefficient of T-L mode were slightly higher than that for L-L mode in (001) BF-CF/STO self-assemble (1-3) structure nano composite could be easily explained. ${ }^{31}$ Although the ME contribution from the magnetostriction for T-L mode ( $\mathrm{d}_{31}$ prefer) is much smaller than that for the L-L mode ( $\mathrm{d}_{33}$ prefer), the ME contribution from magnetic torque for the T-L mode (bending prefer) is much larger than that for the L-L (expanding prefer) mode, which in multi-coupling combination leads to a higher ME effect in the T-L mode than that in the L-L mode.

\section{SUMMARY}

In summary, the physical mechanism of the MET effect was revealed and proved to be huge by a simple MET devices; giant ME coefficients of $100 \mathrm{~V} / \mathrm{cm}$.Oe or $400 \mathrm{nC} / \mathrm{cm}$.Oe at a low (sub-resonant) frequency and a $2100 \mathrm{~V} / \mathrm{cm}$.Oe or $8.4 \mu \mathrm{C} / \mathrm{cm}$.Oe at the first order resonant frequency was achieved. Multicoupling ME effect, which comes from the interaction of magnetostriction, magnetic-torque and piezoelectricity, is taken into the consideration of designing device with the giant ME effect. The construction of giant ME devices have been extended to a broad range of ferromagnetic-piezoelectric systems.

\section{ACKNOWLEDGMENTS}

This research is supported by NSFC (Grant No. 11004207) and Ningbo Natural Science Foundation (Grant No. 2010A610181).

\footnotetext{
${ }^{1}$ M. Fiebig, J. Phys. D 38, R123 (2005).

${ }^{2}$ M. I. Bichurin, V. M. Petrov, and G. Srinivasan, Phys. Rev. B 68, 054402 (2003).

${ }^{3}$ P. Curie, J. Phy. (Paris) 3, 393 (1894).

${ }^{4}$ D. N. Astrov, Sov. Phys. 11, 708 (1960).

${ }^{5}$ B. I. Al'Shin and D. N. Astrov, Sov. Phys. 17, 809 (1963).
} 
${ }^{6}$ T. H. O’Dell, Philos. Mag. 16, 487 (1967).

${ }^{7}$ T. Watanabe and K. Kohn, Philos. Trans. 15, 57 (1989).

${ }^{8}$ B. B. Krichevtsov, V. V. Pavlov, R. V. Pisarev, and A. G. Selitsky, Ferroelectrics 161, 65 (1993).

${ }^{9}$ J. Wang, J. B. Neaton, H. Zheng, V. Nagarajan, S. B. Ogale, B. Liu, D. Viehland, V. Vaithyanathan, D. G. Schlom, U. V. Waghmare, N. A. Spaldin, K. M. Rabe, M. Wuttig, and R. Ramesh, Science 299, 1719 (2003).

${ }^{10}$ J. V. D. Boomgaard, D. R. Terrell, R. A. J. Born, and H. F. J. I. Giller, J. Mater. Sci. 9, 1705 (1974).

${ }^{11}$ J. V. D. Boomgaard and R. A. J. Born, J. Mater. Sci. 13, 1538 (1978).

${ }^{12}$ C. W. Nan, Phys. Rev. B 50, 6082 (1994).

${ }^{13}$ C. W. Nan, M. Li, X. Q. Feng, and S.W. Yu, Appl. Phys. Lett. 78, 2527 (2001).

${ }^{14}$ H. Zheng, J. Wang, S. E. Lofland, Z. Ma, L. Mohaddes-Ardabili, T. Zhao, L. Salamanca-Riba, S. R. Shinde, S. B. Ogale, F. Bai, D. Viehland, Y. Jia, D. G. Schlom, M. Wuttig, A. Roytburd, and R. Ramesh, Science 303, 661 (2004).

${ }^{15}$ J. Ryu, A. V. Carazo, K. Uchino, and H. E. Kim, Jpn. J. Appl. Phys. 40, 4948 (2001).

${ }^{16}$ M. I. Bichurin, V. M. Petrov, and G. Srinivasan, J. Appl. Phys. 92, 7681 (2002).

${ }^{17}$ G. Srinivasan, E. T. Rasmussen, B. J. Levin, and R. Hayes, Phys. Rev. B 65, 134402 (2002).
${ }^{18}$ N. Cai, J. Zhai, C. W. Nan, Y. Lin, and Z. Shi, Phys. Rev. B 68, 224103 (2003).

${ }^{19}$ S. X. Dong, J. F. Li, and D. Viehland, IEEE Trans. Ultrason. Ferroelectr. Freq. Control 51, 793 (2004).

${ }^{20}$ J. Y. Zhai, S. X. Dong, Z. P. Xing, J. F. Li, and D. Viehland, Appl. Phys. Lett. 89, 083507 (2006).

${ }^{21}$ Z. P. Xing, J. F. Li, and D. Viehland, Appl. Phys. Lett. 91, 182902 (2007).

${ }^{22}$ E. Quandt, S. Stein, and M. Wuttig, IEEE Trans. Magn. 41, 3667 (2005).

${ }^{23}$ C. W. Nan, M. I. Bichurin, S. X. Dong, D. Viehland, and G. Srinivasan, J. Appl. Phys. 103, 031101 (2008).

${ }^{24}$ Z. P. Xing, J. F. Li, and D. Viehland, Appl. Phys. Lett. 93, 013505 (2008).

${ }^{25}$ H. Greve, E. Woltermann, H. J. Quenzer, B. Wagner, and E. Quandt, Appl. Phys. Lett. 96, 182501 (2010).

${ }^{26}$ W. Eerenstein, N. D. Mathur, and J. F. Scott, Nature 442, 759 (2006).

${ }^{27}$ J. D. Jackson, Classical Electrodynamics, 3rd ed. (John Wiley \& Sons, New York, 1998), Vol. 5, p. 190.

${ }^{28}$ M. Gürgöze, J. Sound Vibr. 105, 443 (1986).

${ }^{29}$ S. X. Dong, J. Y. Zhai, J. F. Li, and D. Viehland, Appl. Phys. Lett. 89, 252904 (2006).

${ }^{30}$ S. X. Dong, J. F. Li, and D. Viehland, IEEE Trans. Ultrason. Ferroelectr. Freq. Control 50, 1253 (2003).

${ }^{31}$ L. Yan, Z. G. Wang, Z. P. Xing, J. Li, and D. Viehland, J. Appl. Phys. 107, 064106 (2010). 